\title{
Una posible explicación de las posturas a favor y en contra del Matrimonio Igualitario previo a su aprobación en costarricenses del Gran Área Metropolitana
}

\author{
Rafael Román Quirós \\ Universidad de Iberoamérica
}

\section{Resumen}

La presente investigación se ha propuesto explorar el cómo se podría conformar la actitud favorable o desfavorable hacia el matrimonio igualitario (MI) en costarricenses del GAM a partir de un grupo focal virtual y de dos entrevistas llevadas a cabo durante el mes de mayo del 2019, un año previo a su aprobación. La muestra está compuesta de costarricenses mayores de edad y heterosexuales. Las entrevistas individuales abarcaron las experiencias de dos mujeres con perfiles sociodemográficos similares y con posturas opuestas hacia el matrimonio igualitario, las cuales fueron recogidas mediante entrevistas fenomenológicas presenciales, y analizadas bajo el modelo desarrollado con Teoría Fundamentada para el Grupo Focal. De igual forma se realizó el proceso de codificación para ambas entrevistas de manera individual. Como resultado, se obtuvo un modelo de 4 niveles actitudinales que finalmente determinaría la postura a favor o en contra y que se ajusta de paso a los casos individuales de estudio; 1. la caracterización del MI como algo ya sea normal o anormal 2. las consideraciones favorables o desfavorables hacia la conducta homosexual en sí misma 3 . una actitud de peligrosidad hacia el MI y 4. actitudes favorables o desfavorables hacia la regulación legal.

Palabras clave: Matrimonio igualitario, Costa Rica, actitudes, argumentos, grupo focal virtual.

\section{Introducción}

La discusión social sobre el matrimonio igualitario (MI) en Costa Rica, entendido como la unión legal entre dos personas del mismo sexo con el goce de todos los derechos y beneficios de los que gozan las parejas heterosexuales (Andryszewski, 2012), es el resultado de movimientos que tienen su origen en la década de los ochenta con la creación de organizaciones civiles que lucharon por los derechos de las personas pertenecientes a una sexualidad diversa, en medio del pánico ocasionado por el aumento de casos del virus del VIH/SIDA. Durante la década de los noventa, se comienza a problematizar de forma pública la falta de un marco legal para regular este tipo de vínculos, canalizando estas necesidades e intereses a través de distintas instituciones (Jiménez, 2017).

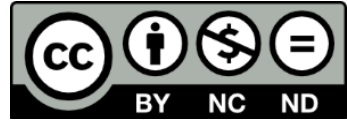


Sin embargo el punto específico del matrimonio entre personas del mismo sexo no será el aspecto prioritario de manera clara en la agenda política de los grupos a favor de los derechos para las personas homosexuales sino hasta las primeras dos décadas del siglo XXI, en las que la Sala Constitucional (creada en 1989 y popularmente conocida como Sala IV) tendría un papel central en cuanto al tema, siendo consultada en diferentes ocasiones (de manera desarticulada) respecto a la inconstitucionalidad de no reconocer estas uniones (Jiménez, 2017).

Tras el triunfo en las pasadas elecciones presidenciales del 2018 del partido oficialista que respaldaba la lucha por los derechos de las personas lesbianas-gais-bisexualestransexuales (LGBT), y tras dos nuevas consultas de constitucionalidad en cuanto al reconocimiento de estas uniones, finalmente la Sala IV dicta sentencia adhiriéndose al fallo del 24 de Noviembre del 2017 de la Corte Interamericana de Derechos Humanos, que exige utilizar las mismas figuras jurídicas ya existentes a las que las personas heterosexuales tienen acceso (Corte Interamericana de Derechos Humanos, 2018), dejando finalmente vigente el matrimonio igualitario en el país desde mayo del 2020.

Los sucesos descritos anteriormente han generado a su vez una importante oposición por parte de ciertos sectores que han encontrado dentro del mismo proceso una representatividad política importante dentro de lo que otrora fue un partido minoritario; de manera en que este no sólo logra disputar la segunda ronda electoral para la presidencia del país, sino que también logra alcanzar una importante representación política parlamentaria (Tribunal Supremo de Elecciones, s.f.).

Es así que la presente investigación se ha propuesto el explorar la conformación de las evaluaciones afectivas en cuanto al matrimonio igualitario en Costa Rica, esto es, la consideración de agradable o desagradable que se establece con cierta estabilidad respecto a objetos sociales, y lo cual constituye el elemento central de las actitudes (Fiske y Taylor, 1991). La actitud como tal es un elemento central de la psicología social desde su inicio (Thomas y Znaniecki, 1918), y por lo tanto ha contado con distintas acepciones a través de las décadas, pasando en un inicio de definiciones amplias que abarcaban dimensiones cognitivas, afectivas, motivacionales y conductuales, a definiciones que hacían un énfasis en la probabilidad de mostrar conductas específicas (Campbell, 1950) y por último a definiciones contemporáneas que circunscriben las actitudes a un componente eminentemente evaluativo (Schwarz y Bohner, 2001), y es en esta última acepción que se entiende la actitud en el presente trabajo, como una tendencia psicológica de evaluación favorable o desfavorable (Eagly y Chaiken, 1993).

En sí, las evaluaciones afectivas o las actitudes componen junto con las emociones y el estado de ánimo lo que genéricamente llamamos Afectividad (Páez y Carbonero, 1993),

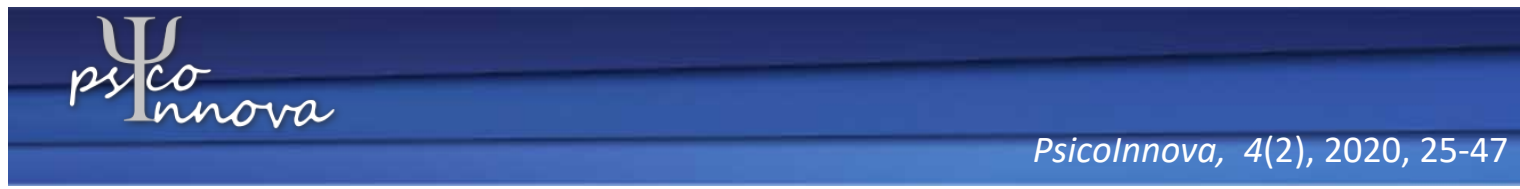


Román-Quirós

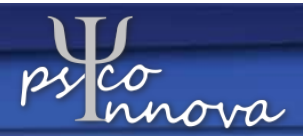

una dimensión o aspecto esencial dentro del ser humano que interactúa con la Cognición.

Dicha interacción entre Cognición y Afectividad fue por mucho tiempo discutida dentro de la disciplina psicológica en cuanto a cuál de ambos elementos se encuentra subordinado al otro o bajo la consideración de que ambos constituyen sistemas con un funcionamiento separado (Páez y Carbonero, 1993). Hoy en día los hallazgos en neurociencias cognitivas indican que contrario a la noción de sistemas separados, las emociones constituyen un componente fundamental e inseparable de la cognición (Lewis, 2005; Pessoa, 2008; Pessoa y Adolphs, 2010). De esta forma, en lugar de oponer ambos sistemas, la evidencia reciente indica que las representaciones cerebrales funcionan de una manera unificada entre emoción, percepción y pensamiento y no como procesos aislados (Todd et al, 2020).

Los estudios en cuanto a actitudes hacia el MI suelen realizarse desde la perspectiva de las creencias culturales y características sociodemográficas (Hodges y Lohan, 2012) de los sujetos, si bien existe evidencia bajo el enfoque de la Teoría del Contacto Social, en el tanto el interactuar con grupos específicos generaría tolerancia hacia los mismos (Pettigrew, 1998). Anderssen (2002) señala que el contacto con personas homosexuales genera actitudes más positivas hacia los mismos, lo que ayuda a que se esté en mayor medida de acuerdo con el MI (Horvath y Ryan, 2003; Pew Research Center, 2003).

Siempre bajo el enfoque cultural y sociodemográfico, la literatura indica que existe un menor apoyo al MI en personas religiosas (Olson, Cadge, y Harrison 2006; Andersen y Fetner 2008; Adamczyk y Pitt 2009; Sherkat et al. 2011; Akker, Ploeg y Scheepers, 2013), en personas que sostienen creencias fundamentalistas (Wilkinson, 2004; Altemeyer y Hunsberger, 1992), de mayor edad (Andersen y Fetner 2008; Adamczyk y Pitt, 2009), en hombres (Wilcox y Wolpert, 2000), en personas casadas o que viven juntas como si lo estuviesen (Díez y Dion, 2018), en personas con un mayor número de hijos (Adamczyk y Pitt, 2009), y en personas que cuentan con círculos significativos en las que un número importante de los contactos son religiosos (Hodges y Lohan, 2012).

Por el contrario, un mayor apoyo al MI se encontraría en personas que manifiestan acuerdo hacia valores democráticos (Dion y Díez, 2017), en personas que habitan en ciudades capitales (Flores, 2014), en hombres y mujeres homosexuales, en personas solteras, con educación universitaria, de mayores ingresos económicos, y en individuos con mayores círculos de educación universitaria (Hodges y Lohan, 2012).

Sin embargo, a pesar de que la información disponible es extensa en países democráticos y desarrollados, resulta escasa en otras latitudes (Brewer, 2014), y resulta aún más escasa para la región latinoamericana (Díez y Dion, 2018), principalmente debido al hecho de que al tratarse de un tema reciente en las diferentes agendas políticas de la región, se contaba con muy pocos datos respecto a dichas posturas hasta hace poco, debido a que

\section{pesco


dicha información no era sondeada por encuestas de opinión; situación que comienza a cambiar a partir del 2010 en la región (Díez, 2015).

De paso, América Latina es un contexto particularmente variado en cuanto a elementos que influirían en actitudes liberales (como los niveles de crecimiento económico, acceso a internet y calidad democrática) los cuales a su vez, influirían en la actitud hacia el MI (Díez y Dion, 2018). Dicho apoyo puede variar desde una aprobación del 18,6\% por parte de la población en El Salvador, a un 35\% para Costa Rica, o inclusive a un 74,6\% en Uruguay (Espinal y Morgan, 2017), razón por la que se hace aún más importante ahondar en la comprensión particular de cada país.

En cuanto a las percepciones que sustentan las actitudes en contra del MI, estas versan primordialmente sobre aspectos biológicos en el sentido de que la homosexualidad no es natural (Sullivan 2006). Los argumentos en oposición han sido estudiados igualmente por Segura (2005), quien señala primordialmente tres aspectos: a) la homosexualidad como algo pecaminoso, perverso y no natural b) una inferioridad natural de estas uniones debido a su incapacidad para concebir y c) que la santidad e importancia de la institución del matrimonio se vería menoscabada minando uno de los fundamentos de la sociedad.

Dentro de las posiciones a favor del MI, se ha encontrado que es más común atribuir la homosexualidad a factores biológicos en lugar de factores de elección personal, además de una tendencia por favorecer la igualdad por sobre la moralidad (Hodges y Logan, 2012). Jiménez (2017) señala que los estudios LGBT consideran que al alcanzar la implementación legal como objetivo, la discriminación hacia las personas homosexuales se vería reducida, ya que la normalización de dicha figura jurídica ayudaría a visualizar a las personas homosexuales como parte de la nación. La anterior consideración se encuentra en consonancia con uno de los elementos esenciales según la Teoría del Contacto Intergrupal de Allport (1954) en el tanto el apoyo de las autoridades, la ley y la costumbre constituye uno de los elementos fundamentales para que la interacción entre grupos tenga efectos positivos (junto con el mismo estatus de los grupos dentro de la situación particular, metas comunes y la cooperación intergrupal). Sin embargo es claro que la lucha por la equiparación de dichas uniones responde también a una serie de necesidades que enfrenta este sector de la población en cuanto al marco legal (herencias, impuestos y beneficios por parte de programas e instituciones sociales). En este sentido, las posturas a favor del MI en América Latina suelen precisamente representarse por parte del activismo como un problema de derechos humanos y de valores democráticos (Díez, 2015).

En el caso costarricense, Smith-Castro y Molina-Delgado (2011) en un estudio que explora las actitudes hacia el MI en Costa Rica, encuentran que una alta ortodoxia religiosa,

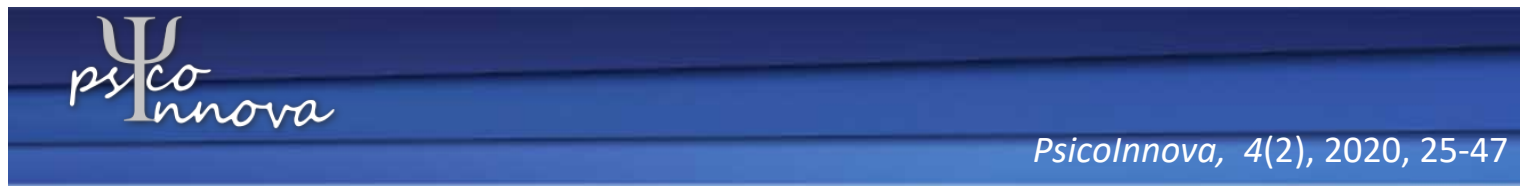




\section{Román-Quirós}

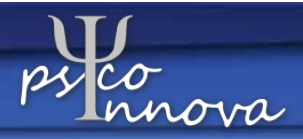

un elevado autoritarismo homofóbico y el poco contacto con personas homosexuales se traducirían en una combinación con bajas probabilidades de apoyo al MI.

En un estudio similar a la presente investigación y también dentro de nuestro contexto, Gonzáles-Suárez (2009) lleva a cabo 94 entrevistas estructuradas en personas a favor y en contra de un proyecto de ley de unión civil entre personas del mismo sexo, encontrando que el tema religioso y la figura de Dios resultaron centrales para los participantes. Así mismo identifica una serie de opiniones, en las cuales se destaca el caracterizar el matrimonio como una institución sagrada, el considerar que el fin primordial de las parejas es el de procrear, así como consideraciones de la sexualidad como una fuente ya sea de amor o de pecado, o una visión de la homosexualidad como algo que propicia el deterioro social.

Sabemos que la cultura de un país no es una entidad estática, sino que este conjunto de ideas, creencias y hábitos está en constante cambio a través del tiempo (Heine, 2016). En este sentido el estudio de Gonzáles-Suárez no sólo fue realizado hace más de diez años, un periodo de tiempo en el cual mucho ha cambiado sobre el tema, sino que además el mismo se realizó con motivo de un proyecto de ley de unión civil, lo que podría no ser equiparable con el MI para una gran parte de la población. De esta manera, la presente investigación se propone dilucidar nuevamente y a la luz de una serie de nuevos acontecimientos que han generado crispación en el país, cuáles podrían ser algunos de los principales argumentos y preocupaciones que nos ayuden a comprender las posturas a favor y en contra durante este momento histórico previo a su aprobación.

\section{Método}

Se parte de un diseño fenomenológico de investigación en el que se emplea la Teoría Fundamentada como método de análisis que permita crear teoría con una consistencia lógica a partir del grupo focal y las entrevistas individuales, enfatizando en la elaboración de núcleos analíticos y códigos a partir de los datos obtenidos, en lugar de trabajar con hipótesis previamente establecidas (Aguilar, Monge, Pérez y Víquez, 2008).

\section{Participantes}

Se contó con la participación de 10 sujetos en total, 9 participantes en el grupo focal y dos mujeres en la década de los cuarenta, sin hijos, del gran área metropolitana, y con estudios superiores en áreas relacionadas a finanzas a las que se les aplicó entrevistas fenomenológicas. Una de estas mujeres participó tanto en el Grupo Focal como en la entrevista individual (en contra). Todos los participantes son costarricenses, usuarios de redes 
sociales, heterosexuales, mayores de edad, y representativos de una postura ya sea a favor o en contra del MI, en el tanto sostenían una postura no instrumental (es decir, estaban en contra o favor por el fondo del tema). Este último punto fue constatado mediante una pregunta incluida en el correo electrónico con el que se estableció el contacto inicial. El consentimiento informado fue adjuntado en el mismo correo electrónico, y en el mismo se especificaron todas las consideraciones éticas del estudio con un formulario oficial del Comité Ético Científico de la Universidad de Costa Rica, solicitándole a los participantes su devolución por este mismo medio con los datos respectivos y haciendo constar el carácter voluntario de su participación, así como la lectura total del documento. Mediante el mismo, se les garantizó de paso el anonimato de los datos proveídos, los cuales serían utilizados únicamente para fines de investigación. Por último, se les solicitó constatar de manera muy puntual si sostenían una postura a favor o en contra del MI para la conformación tanto del Grupo Focal como de las respectivas entrevistas individuales.

En cuanto a los aspectos éticos de la investigación, es importante señalar que la realización de un grupo focal presencial para discutir un tema altamente controversial (Díez y Dion, 2018) podría haber resultado de difícil manejo en su moderación, además de que podría haber indispuesto más de la cuenta a los participantes dentro del grupo de discusión. En este sentido se consideró que una interacción virtual permitiría no sólo el anonimato de los participantes entre sí, sino que además contaría con la continua vigilancia y el control absoluto por parte del investigador (puesto que era posible remover en todo momento cualquier participante o comentario que resultara irrespetuoso) lo cual resultaba más adecuado para salvaguardar la integridad de los participantes en todo momento.

\section{Instrumentos}

Para la realización del grupo focal se utilizó la aplicación y plataforma de mensajería Telegram (http://telegram.com.es/) por varias razones que se explican a continuación. En primer lugar, la aplicación es gratuita y resulta muy familiar para la población costarricense, ya que su interfaz se asemeja mucho a Whatsapp; siendo esta última utilizada por el $83 \%$ de la población; pues es Costa Rica el país latinoamericano que encabeza el uso de la misma (Arias, 2019). Por otro lado, Telegram permite la participación ya sea desde el teléfono móvil o desde una computadora, permitiendo la creación de grupos de discusión con participación anónima siendo que la persona opte por esta modalidad y evitando que los participantes puedan obtener los números telefónicos de otros participantes que están dentro del grupo protegiendo así sus datos. Luego permite al moderador personalizar aquello que le está

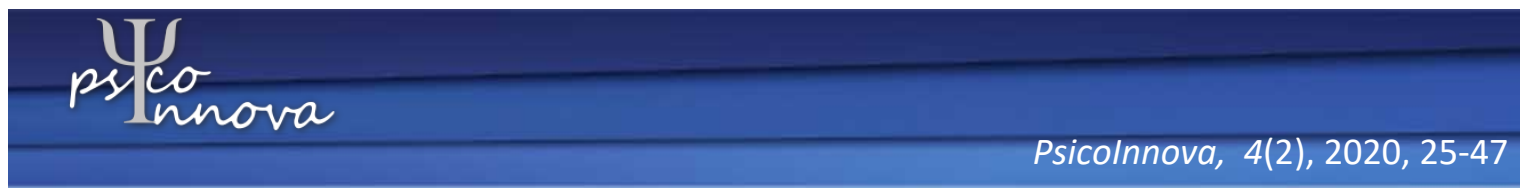




\section{Román-Quirós}

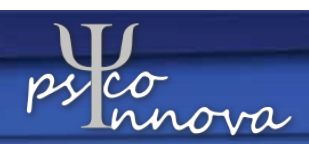

permitido a los participantes hacer, de manera que se puede evitar que estos envíen mensajes, imágenes, video o audio del todo, o cuando se requiera y además de manera separada. Por otro lado permite al moderador sacar participantes en todo momento y borrar comentarios sin que los participantes puedan realizar esto mismo al moderador u otros participantes. Finalmente el registro de datos, se obtuvo a través del historial de la conversación en línea que la plataforma permite.

Por último, se realizaron dos entrevistas fenomenológicas en una persona a favor y otra en contra del MI, para comprender la percepción de ambas durante el proceso de discusión nacional sobre el MI en redes sociales entre enero del 2018 a mayo del 2019, registrando los datos mediante un dispositivo de grabación para su posterior transcripción y análisis.

\section{Procedimientos de recolección}

Al ser contactados por correo electrónico, todos los participantes debieron contestar a una pregunta diseñada para excluir participantes con posturas instrumentales respecto al MI, es decir, con una aprobación o desaprobación por las consecuencias prácticas de la equiparación de estos vínculos y no por el fondo del tema, lo que no sería conveniente en términos de representatividad. La pregunta es: "Ya sea que usted esté a favor o en contra del matrimonio entre personas del mismo sexo; ¿Sostiene usted dicha postura porque considera el fenómeno en sí mismo como algo correcto o incorrecto más allá de cualquier repercusión que pueda tener su aprobación en la práctica?". En ciertos casos en que la pregunta no fue bien entendida por los candidatos, se procedió a reexaminarla con los mismos mediante comunicación telefónica.

En cuanto al grupo focal, se procedió a publicitar la búsqueda de participantes en el grupo de Facebook llamado "Foro Nacional de Costa Rica", un grupo público que para abril del 2019 contaba con más de 1400 usuarios de ambas posturas para lo que se pretendía utilizar el método Snowball. Sin embargo y debido a la limitación de tiempo, se tuvo que proceder a publicar en otros grupos de Facebook similares sin tener éxito, por lo que se procedió a realizar la publicación en las plataformas Reddit y Whatsapp, con lo que se logró 9 participantes, 3 de ellos con posturas en contra.

Una vez que se establecía el contacto inicial, se les enviaba el consentimiento informado, se hacía la recepción del mismo lleno, respondían a la pregunta inicial y se corroboraba que cumplieran con los criterios de inclusión para posteriormente solicitarles bajar Telegram para sus teléfonos celulares. Un día antes de la discusión se constató que todos tuvieran la aplicación debidamente instalada y que aparecieran registrados en la misma.

\section{UTo


Ya que las preguntas del moderador se habrían confundido con los comentarios de los participantes en una discusión que fue intensa y extensa; previendo la situación, se redactaron diferentes preguntas que fueron plasmadas en imágenes que fueran fácilmente notables para todos los participantes (siendo el moderador el único con la potestad de postear imagen alguna).

La discusión tuvo una duración de 1 hora y 15 minutos como fue anunciada previamente, siendo que al final de la misma hubo una despedida y agradecimiento a todos los participantes, bloqueándoles por último la facultad de enviar más mensajes y sacándolos del grupo.

Es importante indicar que es sabido que dentro de los grupos focales presenciales, los participantes experimentan inhibiciones al menos en cuanto a la apariencia física y al presunto estatus social de otros participantes (Fox, Morris y Rumsey, 2007), por lo que la modalidad virtual podría aportar en el sentido de permitir una expresión más libre de las ideas respecto a un tema ya de por sí conflictivo, cuando lo que interesa en este caso particularmente es explorar las argumentaciones detrás de dichas actitudes, las cuales podrían verse inhibidas en la presencialidad. De esta forma, el procedimiento virtual puede ser preferible en ciertos casos (Boydell, Fergie, McDaid y Hilton, 2014) a la vez que los grupos sincrónicos como el de la presente investigación, han reportado resultados similares a los grupos focales presenciales (Stewart y Williams, 2005) así como una calidad de información comparable (Van Eeden-Moorefield et al, 2008).

En el caso de las entrevistas fenomenológicas, se hizo un muestreo a conveniencia buscando a dos mujeres de edades y actividades profesionales similares, en el que una vez que se contaba con el consentimiento informado y que se constataba que la participante cumplía con los criterios de inclusión, se procedía a visitar la casa de habitación en la fecha y hora convenida para las entrevistas que tuvieron una duración aproximada de una hora. Posteriormente se transcribieron las mismas para ser analizadas y los archivos fueron subidos a una nube de almacenamiento con contraseña y eliminados del dispositivo de grabación.

\section{Procedimientos de Análisis}

En cuanto al análisis de los datos, para sistematizar la información se utilizó el software de análisis cualitativo Atlas.ti versión 8.1, en el que bajo la perspectiva epistemológica de la Teoría Fundamentada, si bien se partiría de la revisión de la literatura sobre el tema como un paradigma de codificación, se le daría prioridad a los datos obtenidos en el historial de conversación del Grupo Focal. Durante todo el proceso de análisis se escribió memoranda a partir de las impresiones del investigador al analizar el material.

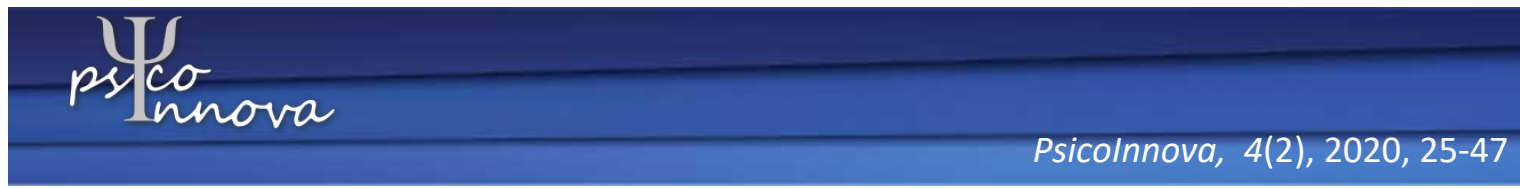


Román-Quirós

peso

unova

Se inició con una codificación abierta en la que se agruparon las unidades

parafraseadas en categorías con propiedades comunes a las que se les asignó un nombre que guardara relación con dichas características. De esta forma se tomaron fragmentos que fueran representativos de argumentos hacia el MI para asignarles un nombre de código que guardara relación con ese contenido particular, siendo que los otros parafraseos que se examinaron a continuación y que contenían características o contenido similar, se les asignó entonces el mismo nombre de código. Posteriormente se agruparon ya no frases, sino unidades conceptuales que reflejaran mejor el fenómeno que se estudia, generando categorías conceptuales más abarcadoras y manejables (Aguilar, Monge, Pérez y Víquez, 2008).

A continuación se procedió a realizar una codificación axial en la que las categorías de la codificación abierta se relacionaron asociando categorías y subcategorías para reensamblar los datos. De esta manera, y de forma cuidadosa, la sistematización de los conceptos fue dando inicialmente un sentido de relación respecto a la actitud hacia el MI. Posteriormente, pero siempre dentro de esta misma fase, se presentaron los vínculos encontrados a través de enunciados relacionales. De esta forma se logró un sistema de categorías y subcategorías supraordenadas y relacionadas (Aguilar et al, 2008).

Luego se integró una explicación que fuera fiel a lo obtenido empíricamente para obtener una proposición teórica que explicara qué contenidos cognitivos podrían sustentar dichas actitudes, seguidas de un refinamiento teórico (Aguilar et al, 2008).

Finalmente se realizó la codificación de las entrevistas fenomenológicas bajo las categorías encontradas en el modelo anterior para evaluar el ajuste entre las actitudes y las experiencias reportadas. Por último, se procedió a realizar las 3 fases descritas anteriormente para ambas entrevistas individuales de forma separada, de forma en que fuera posible analizar comparativamente las codificaciones axiales de los tres conjuntos de datos recolectados.

\section{Resultados}

Del proceso de codificación abierta del grupo focal, se obtuvo un total de 41 subcategorías que fueron organizadas en 8 unidades conceptuales (UC) más comprensivas; todo lo cual refleja una serie de argumentos aparejados a las actitudes emergidas a partir de la codificación axial.

De esta forma, podemos clasificar los diferentes argumentos encontrados en cuanto a su A) temática, B) función y C) estructura. Por la temática que tratan o por el fondo emergieron argumentos 1. Biológicos, 2. Históricos, 3. Teológicos, 4. Legales, 5. Económicos, 6. De salud, 7. Referidos a las características psicológicas en las personas homosexuales, y 8. Axiológicos. Por la función considerada en los diferentes argumentos encontramos: 9. Teleológicos (autorealización, represión) y 10. Políticos (conservadurismo

\section{pstco


y progresismo) y C) por el modo en que están elaborados los argumentos mismos: 11. Esencialistas, 12. Consecuencialistas, 13. Analógicos

\section{Ejemplo 1: argumento (A) temático (1) biológico en el grupo focal}

Tampoco la quimioterapia va a favor del orden natural. Ahora, si por natural trata de descalificar a la homosexualidad: ella está presente en decenas de especies. Mas natural... [Errores del original]

\section{Ejemplo 2: argumento (B) funcional (9) teleológico en entrevista individual}

"todo va a ser temporal, entonces yo me tengo que ir preparando para esa eternidad, entonces si yo aquí en esta vida vivo dándole gusto a todo lo que la carne me pide, y dejo de lado lo que mi espíritu me muestra que es lo correcto, lo que me da paz, lo que me da seguridad, verdadera seguridad, porque hacer lo que a Dios le agrada, es lo que a mí me va a dar esa seguridad." [Errores del original]

En el ejemplo 2 podemos observar la concepción respecto a la autorrealización personal por parte de la entrevistada, en el tanto la finalidad o el sentido se alcanza a través de una espiritualidad que implica reprimir los deseos sexuales desviados de la norma.

\section{Ejemplo 3: argumento (C) estructural (11) esencialista en el grupo focal Jesucristo es amor!! Amor es amor de cualquier forma [Errores del original]}

\section{Codificación axial del grupo focal}

En cuanto a la codificación axial, primeramente se procedió a realizar un esquema general mediante la función de red del Atlas.ti que permitiera analizar de forma gráfica las interrelaciones entre unidades conceptuales y las categorías abiertas, para posteriormente hacer una examinación cuidadosa de los contenidos conceptuales de dichas categorías y subcategorías, para poder establecer cuáles unidades conceptuales podrían ser agrupables bajo diferentes categorías axiales.

De esta manera, emergieron 4 categorías axiales; actitudes en este caso, que explicarían la postura hacia el MI, cada una compuesta a su vez por 2 unidades conceptuales, a saber: 1. La actitud de normalidad hacia el MI, la cual incluye las unidades conceptuales "Naturalidad" y "Concepto matrimonial"; 2. La actitud respecto a la conducta homosexual, la cual incluye las unidades "Salud mental" y "Moralidad"; 3. La actitud respecto a la

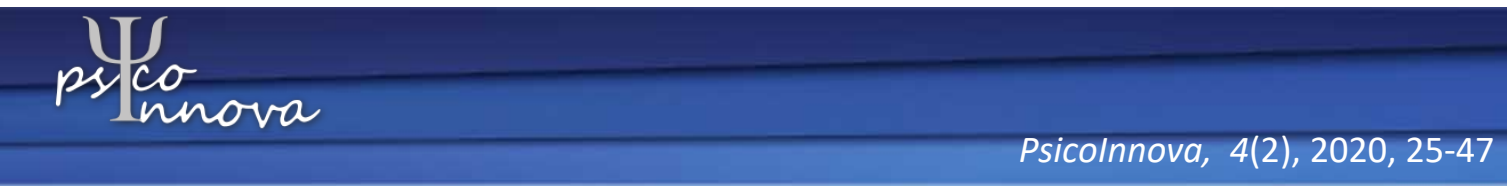


Román-Quirós

peligrosidad del MI que incluye "Daño social" y "Persona homosexual" y 4. La actitud hacia la regulación del MI que incluye "Protección" y "Legitimidad".

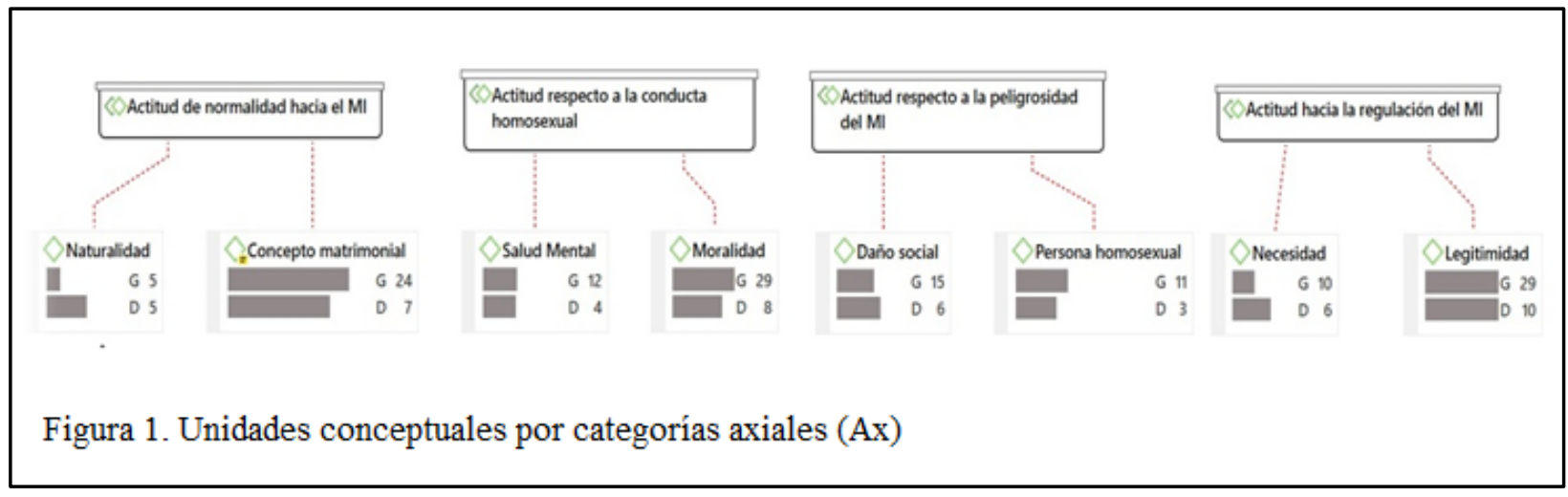

\section{Enunciados relacionales generales del modelo}

Se plantea que los argumentos que componen las 8 unidades conceptuales (UC) reflejan las 4 actitudes resultantes del proceso de codificación axial, en el sentido en que consta en la figura 1, influyendo finalmente en la postura a favor o en contra del MI.

Es decir, que en el caso concreto de la actitud de normalidad hacia el MI, la misma estaría determinada por el conjunto de argumentos que versan sobre a) las finalidades a las que deben tender las vinculaciones afectivas y sexuales (Naturalidad) y b) aquellas respecto a qué se debería entender por matrimonio, (Concepto matrimonial). De esta manera, la actitud respecto a la normalidad del MI podría consistir en un primer nivel dentro de un continuo.

De esta forma, en un segundo nivel actitudinal tendríamos las consideraciones respecto a qué es lo correcto en combinación con aquellas actitudes respecto a qué debe entenderse por estar mentalmente sano, lo que determinaría las Actitudes hacia las relaciones homosexuales en sí (como algo de agrado o desagrado).

El tercer nivel abarcaría consideraciones de lesividad o daño social relacionadas a la instauración (o no instauración) del MI, aunado a la caracterización de la persona homosexual, lo cual nos indicaría la Actitud de peligrosidad hacia estas uniones.

Por último, en un cuarto nivel actitudinal, contaríamos con los argumentos respecto a si es necesario o no regular estas relaciones en combinación con los argumentos en cuanto a qué es lo que provee de fundamento a la ley para que esta lo sea, lo que nos indicaría las actitudes de las personas hacia la regulación.

Es entonces que las consideraciones respecto a 1 . Si la normalidad es o no favorable, 2. Si las relaciones homosexuales son positivas ó negativas, 3. Si estas dañan o no a la sociedad y 4. La necesidad-innecesariedad de regularlas; aquello que podría explicar finalmente el proceso por el cual se establece una postura respecto al MI (a favor o en contra).

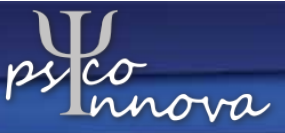




\section{Tabla 1}

Definiciones y parafraseos por unidad conceptual (UC)

\begin{tabular}{|c|c|c|}
\hline & Parafraseos & Definición \\
\hline 1. Moralidad & 29 & $\begin{array}{l}\text { Consideraciones respecto a cómo debe determinarse } \\
\text { lo bueno y lo malo. }\end{array}$ \\
\hline 2. Legitimidad & 29 & $\begin{array}{l}\text { Nociones respecto a aquellos aspectos que proveen } \\
\text { a las leyes de fundamento. }\end{array}$ \\
\hline $\begin{array}{l}\text { 3. Concepto } \\
\text { matrimonial }\end{array}$ & 24 & $\begin{array}{l}\text { Abarca todas aquellas concepciones respecto a qué } \\
\text { debe entenderse por "matrimonio". }\end{array}$ \\
\hline 4. Daño social & 15 & $\begin{array}{l}\text { Consideraciones respecto a la lesividad o los } \\
\text { problemas que la conducta pueda causar en la } \\
\text { sociedad. }\end{array}$ \\
\hline 5. Salud mental & 12 & $\begin{array}{l}\text { Consideraciones respecto a qué debe entenderse por } \\
\text { "salud mental". }\end{array}$ \\
\hline $\begin{array}{l}\text { 6. Persona } \\
\text { homosexual }\end{array}$ & 11 & Caracterizaciones sobre las personas homosexuales. \\
\hline 7. Protección & 10 & $\begin{array}{l}\text { Argumentaciones respecto a si la figura del MI } \\
\text { requiere o no, ser instaurada. }\end{array}$ \\
\hline
\end{tabular}


Román-Quirós

8. Naturalidad

Percepciones que proveen una comprensión

5 teleológica respecto a la vinculación sexual y afectiva de las personas.

La Tabla 1 da cuenta de cuáles fueron los temas que dominaron la discusión en el grupo focal, evidenciando que las categorías de moralidad y legitimidad, se constituyeron como las categorías centrales.

\section{Ajuste del modelo encontrado en el Grupo Focal respecto a las entrevistas individuales}

Si bien es cierto, el modelo propuesto a partir del grupo focal podría hacer pensar que las actitudes de peligrosidad son un nivel prescindible para las posturas a favor (puesto que quien percibe la homosexualidad como algo positivamente normal no presentaría consideraciones de peligro), se encontró por el contrario una fuerte preocupación en la entrevistada en el sentido de lo que ocurriría de no aprobarse el MI:

\section{Ejemplo 4: Daño social (argumento de tipo consecuencialista), entrevista individual \\ "usted no sabe el nivel terror que yo tenía de que esto pasara; porque yo sentía que era como lo mismo que estaba pasando en Estados Unidos (.5) o ahorita en Brasil, (.5) ósea personas gobernando con una posición muy extrema, y muy extrema en contra de los derechos humanos, es que ese es el asunto."}

Basado en lo anterior, los 4 niveles parecerían también ser relevantes en las posturas a favor, lo que indicaría que hay ajuste al modelo propuesto. En la entrevista individual de la persona en oposición, se evidencia una consideración de 1) la homosexualidad como algo antinatural (por lo que en este caso "normal" sería lo positivo y lo establecido por Dios), 2) una caracterización de la conducta como una desvalorización y degeneración sexual personal, 3) una consideración de estos vínculos como peligrosos (es en la entrevista individual en donde aparece el argumento del menoscabo de la familia como socavamiento social, la adopción de niños y la aparición de "todo tipo de enfermedades") y 4) una actitud respecto al no legitimar dichos vínculos de los cuales la sociedad debe ser protegida en el tanto representan una amenaza. De esta forma, el modelo se ajusta en el sentido de que los 4 niveles actitudinales se encuentran también presentes en los casos individuales. 


\section{Comparación entre las codificaciones axiales de las fuentes de datos}

Tras la codificación individual de cada entrevista fenomenológica (de forma separada), en la entrevista a favor se obtuvo un total de 5 unidades conceptuales y tres categorías axiales: A) una actitud de aceptación social B) una actitud de peligrosidad (ante la no aprobación) y C) una actitud positiva ante la regulación a favor de los derechos de las personas homosexuales, siendo la actitud de peligrosidad, lo que explicaría el paso de la aceptación a la protección de este grupo minoritario.

De la entrevista en contra emergen 9 unidades conceptuales y 4 categorías axiales; A) actitudes respecto a la autorealización individual B) actitudes respecto a la moralidad C) actitudes respecto a la peligrosidad del MI y D) Actitudes respecto a la legitimidad.

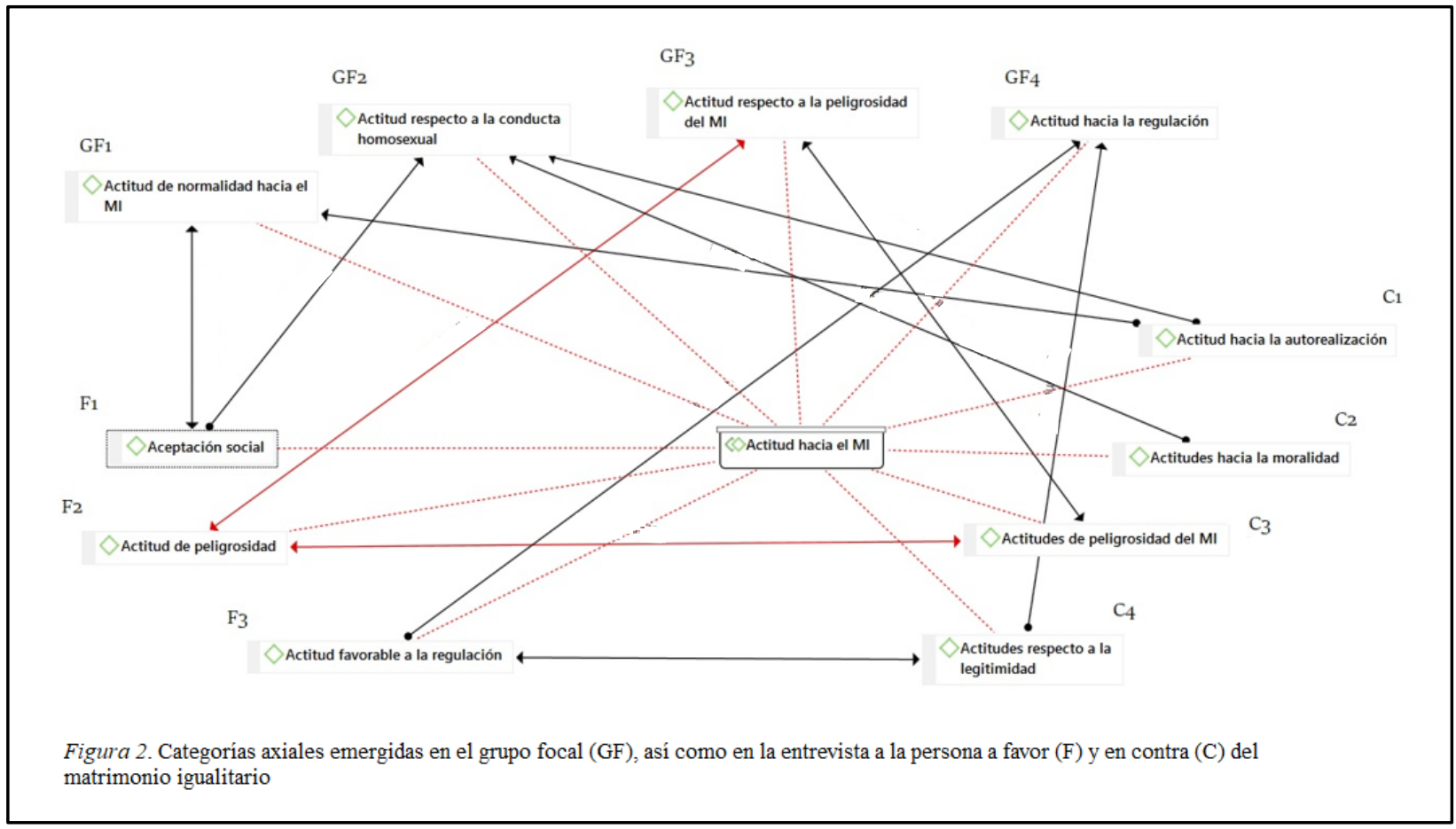

Al comparar las codificaciones axiales de las tres fuentes de datos, encontramos que si bien es cierto la actitud respecto a la normalidad del MI (Grupo Focal) no se presenta con este nombre específico en las 3 categorías axiales obtenidas en la entrevista a favor, la aceptación de este grupo minoritario constituiría una actitud favorable para las dos acepciones de normal/anormal en este caso; esto es, tanto la aceptación hacia lo anormal (o contrario a la norma en un sentido de frecuencia) como la aceptación hacia el hecho de que

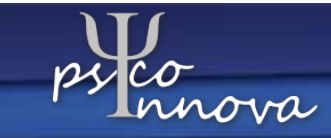




\section{Román-Quirós}

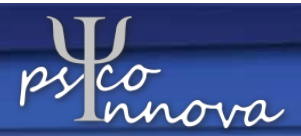

estos vínculos son también normales (en el sentido de ser algo de natural ocurrencia entre los seres humanos).

De igual forma, tampoco ha emergido una categoría del mismo nombre en la codificación axial de la entrevista en contra, sin embargo es deducible a partir de diferentes comentarios externados en la misma entrevista, en el tanto que "normal" es para la entrevistada, aquello que ha sido establecido por Dios, siendo lo anormal su opuesto (con una connotación respectivamente negativa).

\section{Ejemplo 5:}

El aceptar las uniones homosexuales y legalizarlas, va a traer (...) que la gente se abra, se abra completamente a que esto es normal y natural. Entonces se va a desnaturalizar. El ser humano se va a ir desnaturalizando cada vez más. Y entonces cuando un ser humano se desnaturaliza, vienen muchas consecuencias (...) a nivel moral, y a nivel social. Me preocupa el hecho de que la familia, de que el plan inicial para la familia de Dios, pues no... Con este tipo de uniones no se está llevando a cabo. Y entonces, ¿qué es lo que pasa? Que, el mandato de Dios y el sueño de Dios con crear al hombre y a la mujer para que los dos se unieran en matrimonio y formaran una sola carne y se multiplicaran, procrearan, y el sentido de la familia cristiano, se pierde con este tipo de uniones, verdad [errores gramaticales de origen].

Es decir, considerar el MI como positivo y normal ó por el contrario como negativo y anormal es algo que estuvo presente en ambas entrevistas, si bien la entrevistada en contra ahondó profusamente y en mayor medida en consideraciones de qué consideraba como autorealización (felicidad verdadera) y de qué forma podían las personas homosexuales alcanzarla, razón por la cual este tema terminó siendo una categoría axial. Sin embargo es evidente que el primer nivel actitudinal del modelo se hace presente en ambos casos.

El segundo nivel actitudinal referido a la caracterización de la conducta homosexual, se encuentra también presente de manera implícita en ambas entrevistas, puesto que encontramos la aceptación de dichos vínculos en una y la caracterización de los mismos como sexualmente degradantes y deshumanizantes en la otra entrevista. Es aquí en donde encontramos un discurso que parece clave dentro de la teoría, en el tanto el concepto "degradación" parece ser el inicio de un proceso que permite pasar de consideraciones negativas de la conducta homosexual, a consideraciones de la homosexualidad como algo socialmente peligroso.

\section{Ejemplo 6: Degradación (argumento de tipo axiológico), grupo focal}

"Precisamente la ignorancia a los preceptos divinos lleva al hombre a degradarse"

\section{pesco


"Degrada el plan original de Dios, degrada la familia, degrada la salud, degrada la procreación, degrada al ser humano"

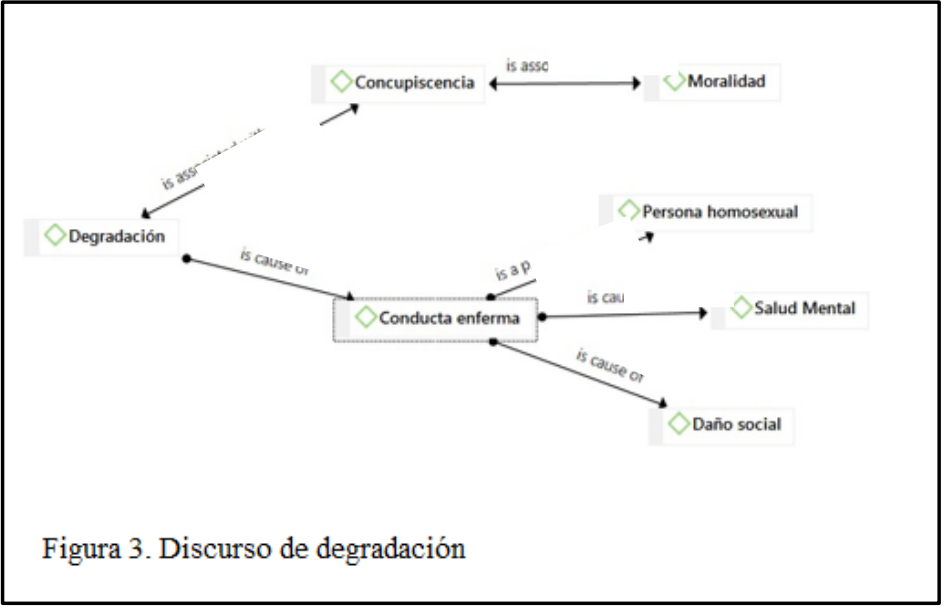

De esta forma se acabaría por considerar a la conducta homosexual como una conducta enferma, considerando implícitamente al homosexual como una persona enferma que contagiará a otros causando un daño social.

\section{Ejemplo 7: Conducta enferma (argumento de tipo analógico)}

"Ahora si a mi se me ocurre que yo puedo establecer relaciones de pareja con animales voy a la corte de Derechos Humanos" [errores del original]

Se había mencionado anteriormente que existe evidencia de que perspectivas positivas de las persona homosexuales tienen relación con posturas más positivas hacia el MI (Horvath y Ryan, 2003; Pew Research Center, 2003). Esto da pie a pensar en su contrario, esto es que perspectivas más negativas hacia las personas homosexuales, tendría relación con posturas más negativas hacia el MI, siendo que la consideración de esta figura jurídica como algo socialmente peligroso, constituye una de sus manifestaciones. De hecho la peligrosidad está presente en todas las codificaciones axiales como elemento previo a las consideraciones respectivas de legitimidad y legalidad, últimas que en la entrevista a favor representan una variedad de principios generales del derecho (sobretodo la separación entre el derecho y la religión y la noción de garantías individuales), mientras que en la entrevista en contra se cuenta con una comprensión de la legitimidad de tipo teocéntrico. De esta forma se concluye que el modelo se encuentra también presente en ambas entrevistas individuales.

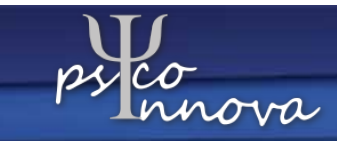




\section{Román-Quirós}

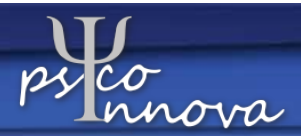

Por último y como un núcleo central claro en la entrevista en contra se encuentra

"Salud mental", una categoría que cuenta con múltiples argumentos a favor de la represión

de conductas sexuales desviadas de la norma como única vía de autorrealización. Como categoría central en la entrevista a favor encontramos argumentos a favor de la condición de igualdad, lo que emerge en el mismo sentido de la literatura, en el tanto existiría una mayor tendencia a favorecer la igualdad por sobre la moralidad en las personas a favor del MI (Hodges y Logan, 2012).

\section{Conclusiones}

La presente investigación se ha propuesto explorar el cómo se conformaría la actitud favorable o desfavorable hacia el matrimonio igualitario en costarricenses del GAM durante el mes de mayo del 2019, es decir, un año antes de su implementación. Tras el análisis de un Grupo Focal y de dos entrevistas individuales a la luz de la Teoría Fundamentada, se propone una teoría que supone un modelo de cuatro actitudes secuenciales dentro de la conformación final favorable o desfavorable hacia el MI.

En un primer momento o nivel, contaríamos con una caracterización del MI como algo ya sea normal o anormal. En este sentido, la consideración del MI como algo anormal (sea desde una perspectiva natural o estadística) por sí sola, no es necesariamente indicativa de una actitud desfavorable hacia el MI como tal (puesto que el participante podría también considerar que dichos vínculos no son usuales o que son fenómenos de minorías, sin que el mismo desapruebe necesariamente dichas uniones).

En un segundo nivel actitudinal, tendríamos las consideraciones favorables o desfavorables hacia la conducta homosexual en sí misma (sin tomar en cuenta la institución del matrimonio), y dentro de los cual entran en juego consideraciones respecto a la salud mental y la moralidad de dichos comportamientos.

En un tercer nivel tendríamos una actitud de peligrosidad hacia el MI, en el tanto dicha peligrosidad se incline para unos o para otros ya sea hacia el peligro que representa para estos sectores minoritarios el que el MI no sea aprobado en el país en el estado actual de las cosas (es decir, con fuerzas políticas que externan y legitiman actitudes discriminatorias, y que eventualmente podrían llegar a tener puestos de poder), ó hacia el peligro de su aprobación por el daño social que causaría. En este último sentido se evidencia la magnitud de significado que tiene la aprobación del MI para la participante en contra, en el tanto se trata de un agravio abstracto y extendido a muchas dimensiones de la convivencia social, frecuentemente expresado a través de la palabra “degradación”. Da la impresión de que la vivencia de dicha participante podría bien ser representada por el significado que atribuye la Real Academia Española a la palabra "concupiscencia" (figura 3), esto es, un

\section{$\bigcup_{0}$


deseo de placeres sexuales desordenados y deshonestos (2019). Si el término degradación nos remite a la noción de un "valor"; concupiscencia y conducta enferma nos remiten a "sexualidad" y "enfermedad" respectivamente. Es decir, se trata de una consideración de la sexualidad como algo de valor que al menoscabarse, es sujeto de contagio; una interesante discusión desde una perspectiva filosófica e histórica, pues finalmente el MI es un tema que tiene a la sexualidad de por medio.

En un cuarto nivel, contamos con actitudes favorables o desfavorables hacia la regulación legal para dichas uniones; si bien es cierto que el MI implica no una regulación legal, sino una equiparación de derechos que es distinto. En este sentido, aquello que es favorable dentro del cuarto nivel abarca ambos aspectos, si bien dentro del grupo de discusión se contó con una participante que se oponía a la equiparación de estos vínculos con los heterosexuales reconociendo a su vez la necesidad de proveerlos de un marco legal. Llama la atención en este último sentido la existencia de una discusión de fondo en torno a qué es lo que le da legitimidad a una ley (¿la voluntad de la mayoría, el derecho natural, derechos humanos, o la concordancia de las normas con los textos sagrados?), algo de sumo interés para la Filosofía del Derecho, en el tanto estas nociones podrían inclinar finalmente la balanza en la postura a favor o en contra del MI. Es importante resaltar que tanto el tema de la moralidad de la conducta homosexual, como la legitimidad de las leyes que vendrían a regular estos vínculos fueron ambos los aspectos más discutidos dentro del Grupo Focal contando cada aspecto con 29 parafraseos respectivamente.

En cuanto al modelo teórico propuesto a partir del Grupo Focal, este no sólo se ajustó a las entrevistas individuales, sino que también resultó similar a las categorías emergidas a partir de la codificación individual de las entrevistas. Por último, al tratarse de un grupo de discusión respecto a un tema controvertido que desata pasiones en la ciudadanía y en el que se encontraban participantes con posturas antagónicas dentro del mismo, se considera que la utilización de una metodología virtual fue un acierto en cuanto a la calidad de la información recolectada, lo cual se refleja en la variedad de acotaciones argumentales encontradas. Las diferentes tipologías se clasificaron en a) argumentos temáticos b) funcionales y c) estructurales. Los temas argumentales fueron 1. Biológicos, 2. Históricos, 3. Teológicos, 4. Legales, 5. Económicos, 6. De salud, 7. Referidos a las características psicológicas en las personas homosexuales, 8. Axiológicos, 9. Teleológicos (respecto a la autorealización, y la represión), y 10. Políticos (conservadurismo y progresismo).

Finalmente, la inclusión de participantes con posturas en contra resultó difícil, quizás por el contexto en que se encontraba el país en dicho momento, aunado a que la presente investigación representa un esfuerzo por parte de un actor académico; un sector que podría percibirse por dichos sectores como juez y parte contraria a sus valores e intereses. Por esta

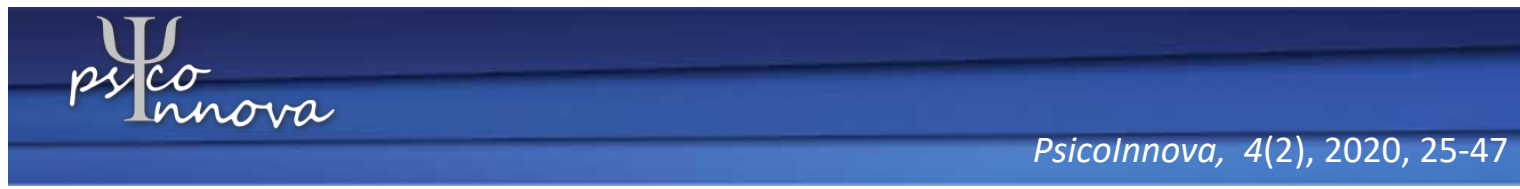




\section{Román-Quirós}

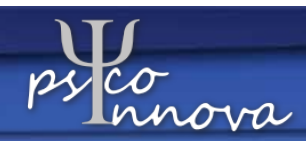

misma dificultad, una misma participante fue parte tanto del Grupo Focal como de la entrevista individual en contra, lo que representa una limitación al presente estudio, en el tanto la misma sostenía una postura religiosa que se consideraría fundamentalista y no coincidente con los otros participantes en contra del MI, quienes ajustándose a lo encontrado en la literatura, se centraron en aspectos no ya teológicos como la participante referida anteriormente, sino biológicos (la homosexualidad como algo que es negativo ya que no es la norma dentro de la naturaleza) (Sullivan, 2006) y recurriendo los mismos a uno de los principales argumentos presentados por Segura (2005), en tanto a una inferioridad natural de las uniones homosexuales por su incapacidad de concebir, presentando a la procreación como la finalidad del vínculo matrimonial, haciendo pasar el eje central de la normalidad por la comprensión del matrimonio a partir de su finalidad. Los otros dos tipos de argumentaciones encontradas por Segura (2005) para las personas en contra; esto es, la homosexualidad como algo pecaminoso y el menoscabo de la familia como elemento central de la sociedad, fueron más bien argüidos de manera exclusiva por la participante con creencias fundamentalistas.

Por último y para futuras investigaciones, sería deseable abordar la temática de forma individual y a mayor profundidad en hombres, en especial tratándose de un tema relacionado a la sexualidad.

\section{Referencias}

Adamczyk, A. Y Pitt, C. (2009). Shaping Attitudes about Homosexuality: The Role of Religion and Cultural Context. Social Science Research, 38(2): 338-351. DOI: https://doi.org/10.1016/j.ssresearch.2009.01.002

Allport, G. W. (1954). The Nature of Prejudice. Reading, M. A.: Addison-Wesley.

Aguilar, W., Monge, D., Pérez, R. Y Víquez, D. (2008). La opción de los métodos de comparación constante en psicología [Serie Cuadernos Metodológicos]. Instituto de Investigaciones Psicológicas, Universidad de Costa Rica. Recuperado de http://www.iip.ucr.ac.cr/es/publicaciones/serie-cuadernos-metodologicos

Arias, J. P. (3 de abril del 2019). Costa Rica lidera uso de Whatsapp y Facebook en Latinoamérica. CrHoy. Recuperado de https://www.crhoy.com/tecnologia/costarica-lidera-uso-de-whatsapp-y-facebook-en-latinoamerica/

Akker, H., Rozemarijn, P., y Peer, S. (2013). Disapproval of Homosexuality: Comparative Research on Individual and National Determinants of Disapproval of Homosexuality in 20 European Countries. International Journal of Public Opinion Research, 25(1): 64-86. DOI: https://doi.org/10.1093/ijpor/edr058 
Anderssen, N. (2002). Does contact with lesbians and gays lead to friendlier attitudes? A two year longitudinal study. Journal of Community and Applied Social Psychology, 12, 124 -136. DOI: https://doi.org/10.1002/casp.665

Andersen, R. y Fetner, T. (2008). Economic Inequality and Intolerance: Attitudes toward Homosexuality in 35 Democracies. American Journal of Political Science, 52 (4), 942-958. DOI: https://doi.org/10.1093/ijpor/edr058

Andryszewski, T. (2012). Same-Sex Marriage: Granting Equal Rights or Damaging the Status of Marriage? Minneapolis, MN: Twenty-First Century Books. Recuperado de http://search.ebscohost.com/login.aspx?direct=true\&db=nlebk\&AN=386716\&lang= es\&site $=$ ehost-live

Boydell, N., Fergie, G., McDaid, L., y Hilton, S. (2014). Avoiding Pitfalls and Realising Opportunities: Reflecting on Issues of Sampling and Recruitment for Online Focus Groups. International Journal of Qualitative Methods, 13(1), 206-223. https://doi.org/10.1177/160940691401300109

Brewer, P. R. (2014). Public Opinion About Gay Rights and Gay Marriage. International Journal of Public Opinion Research, 26(3): 279-282. DOI: https://doi.org/10.1093/ijpor/edu029.

Campbell, D. T. (1950). The indirect assessment of social attitudes. Psychological Bulletin, 47, 15-38. DOI: http://dx.doi.org/10.1037/h0054114

Corte Interamericana de Derechos Humanos. (2018). [Comunicado] Opinión Consultiva sobre identidad de género y no discriminación a parejas del mismo sexo. Recuperado de http://www.corteidh.or.cr/docs/comunicados/cp 01 18.pdf

Díez, J. (2015). The Politics of Gay Marriage in Latin America: Argentina, Chile and Mexico. New York: Cambridge University Press. DOI:

https://doi.org/10.1017/CBO9781316162804

Díez, J., y Dion, M. L. (2018). New Media and Support for Same-Sex Marriage. Latin American Research Review, 53(3), 466-484. https://doi.org/10.25222/larr.345

Dion, M. y Díez, J. (2017). Democratic Values, Religiosity, and Support for Same-Sex Marriage in Latin America. Latin American Politics and Society, 59(4): 75-98. DOI: https://doi.org/10.1111/laps.12034

Eagly, A. H., y Chaiken, S. (1993). The psychology of attitudes. Fort Worth, TX: Harcourt Brace Jovanovich. DOI: https://doi.org/10.1002/mar.4220120509

Espinal, R. y Morgan, J. (2017). Cultura política de la democracia en la República Dominicana y en las Américas, 2016/2017: Un estudio comparado sobre democracia $\quad y \quad$ gobernabilidad. Recuperado de 


\section{Román-Quirós}

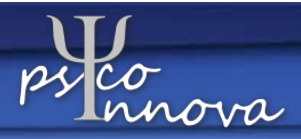

https://WwW.vanderbilt.edu/lapop/dr/AB2016-

17_Dominican_Republic Country_Report W_12.11.17.pdf

Fiske, S. T. y Taylor, S. E. (1991). Social Cognition. New York: McGraw Hill, Inc.

Flores, A. (2014). Reexamining Context and Same-Sex Marriage: The Effect of Demography on Public Support for Same-Sex Relationship Recognition. International Journal of Public Opinion Research, 26(3):283-300. DOI: https://doi.org/10.1093/ijpor/edu020

Fox, F. E., Morris, M., y Rumsey, N. (2007). Doing synchronous online focus groups with young people: Methodological reflections. Qualitative Health Research, 17(4), 539_ 547. Doi:10.1177/1049732306298754

Heine, S. J. (2016). Cultural Psychology. (3 ed). New York:W. W. Norton and Company

Hodges, M. I., y Logan, C. D. (2012). Psychology of Attitudes. Hauppauge, N.Y.: Nova Science Publishers, Inc. Recuperado de: http://search.ebscohost.com/login .aspx?direct=true \&db=nlebk\&AN=541156\&lang= es\&site=ehost-live

Horvath, M., y Ryan, A. M. (2003). Antecedents and potential moderators of the relationship between attitudes and hiring discrimination on the basis of sexual orientation. Sex Roles, 48, 115 -130. DOI: https://doi.org/10.1023/A:1022499121222

Jiménez Bolaños, J. (2017). Matrimonio Igualitario en Costa Rica: Los orígenes del debate 1994-2006. Revista de Ciencias Sociales, 155 (1), 157-172. Recuperado de https://revistas.ucr.ac.cr/index.php/sociales/article/view/30261

Lewis, M. D. (2005). Bridging emotion theory and neurobiology through dynamic systems modeling. The Behavioral and Brain Sciences, 28(2), 169-194. DOI: $10.1017 / \mathrm{s} 0140525 \mathrm{x} 0500004 \mathrm{x}$

Olson, Laura R., Wendy Cadge, y James T. Harrison. (2006). Religion and Public Opinion about Same-Sex Marriage. Social Science Quarterly, 87 (2): 340-360. DOI: https://doi.org/10.1111/j.1540-6237.2006.00384.x

Páez, D., y Carbonero, A. (1993). Afectividad, cognición y conducta social. Psicothema, 5, 133-150.

Pessoa, L. (2008). On the relationship between emotion and cognition. Nature Reviews Neuroscience, 9, 148-158. https://doi.org/10.1038/nrn2317

Pessoa, L. y Adolphs, R. (2010). Emotion processing and the amígdala: from a low road to many roads of evaluating biological significance. Nature Reviews Neurosicence, 11, 773-783. doi: $\underline{10.1038 / n r n 2920}$ 
Pettigrew, T. F. (1998). Intergroup contact theory. Annual Review of Psychology, 49, 65-85.

Pew Research Center for the People and the Press. (2003). Republicans unified, Democrats split on gay marriage : religious beliefs underpin opposition to homosexuality. Recuperado de http://people - press.org/report/197/religious -beliefs -underpin opposition -to- homosexuality.

Real Academia Española. (2019). Concupiscencia. Recuperado de: https://dle.rae.es/concupiscencia

Segura, G. M. (2005). A Symposium on the Politics of Same-Sex Marriage-An Introduction and Commentary. PS: Political Science \& Politics, 38(2), 189-193. DOI: https://doi.org/10.1017/S1049096505056283

Schwarz, N. y Bohner, G. (2001). Blackwell handbook of social psychology: intraindividual processes. Recuperado de https://onlinelibrary.wiley.com/doi/book/10.1002/9780470998519

Sherkat, D. E., Powell-Williams, M., Maddox, G. Y de Vries, K. M. (2011). Religion, Politics, and Support for Same-Sex Marriage in the United States, 1988-2008. Social Science $\quad$ Research $\quad 40(1)$ 167-180. DOI: https://doi.org/10.1016/j.ssresearch.2010.08.009

Smith-Castro, V., y Molina-Delgado, M. (2011). Actitudes hacia el matrimonio y la unión civil gay en Costa Rica:¿ religiosidad, homofobia, autoritarismo o desconocimiento?. Interamerican Journal of Psychology, 45(2), 133-143.

Stewart, K., y Williams, M. (2005). Researching online populations: The use of online focus

groups for social research. Qualitative Research, 5(4), 395-416.

DOI:10.1177/1468794105056916

Gonzáles-Suarez, M. (2009). Proyecto de Ley de Unión Civil entre personas del mismo sexo. Posiciones a favor y en contra. Wimblu, 4(1), 11-30. https://revistas.ucr.ac.cr/index.php/wimblu/article/view/1587

Sullivan Blum, C. R. (2006). "The Natural Order of Creation": Naturalizing Discourses in the Christian Same-Sex Marriage Debate. Anthropologica, 48(2), 203-215. DOI: https://doi.org/10.2307/25605311

Thomas, W. I., y Znaniecki, F. (1918). The Polish peasant in Europe and America. Vol. 1. Boston, MA: Badger. 
Román-Quirós

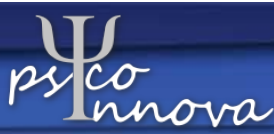

Todd, R. M., Miskovic, V., Chikazoe, J. y Anderson, A. K. (2020). Emotional Objectivity: Neural representations of emotions and their interaction with cognition. Annual Review of Psychology, 71, 25-48. https://doi.org/10.1146/annurev-psych-010419051044

Tribunal Supremo de Elecciones. (s.f.). Estadísticas de procesos electorales. https://www.tse.go.cr/estadisticas_elecciones.htm

Van Eeden-Moorefield, B., Proulx, C. M., y Pasley, K. (2008). A comparison of internet and face-to-face (FTF) qualitative methods in studying the relationships of gay men. Journal

of GLBT Family Studies, 4(2), 181-204. DOI:10.1080/15504280802096856

Wilcox, C. y Wolpert, R. (2000). Gay Rights in the Public Sphere: Public Opinion on Gay and Lesbian Equality. The Politics of Gay Rights [409-432]. Chicago: University of Chicago Press.

Wilkinson, W. W. (2004). Religiosity, authoritarianism, and homophobia: A multidimensional approach. The International Journal for the Psychology of Religion, 14(1), 55-67. Recuperado de: http://dx. Doi. Org/10. 1207/s15327582ijpr1401_5 